\title{
Bionic lightweight design by laser additive manufacturing (LAM) for aircraft industry
}

Claus Emmelmann, Maren Petersen, Jannis Kranz, Eric Wycisk

Claus Emmelmann, Maren Petersen, Jannis Kranz, Eric Wycisk, "Bionic lightweight design by laser additive manufacturing (LAM) for aircraft industry," Proc. SPIE 8065, SPIE Eco-Photonics 2011: Sustainable Design, Manufacturing, and Engineering Workforce Education for a Green Future, 80650L (29 April 2011); doi: 10.1117/12.898525

SPIE. Event: SPIE Eco-Photonics, 2011, Strasbourg, France 


\title{
Bionic Lightweight Design by Laser Additive Manufacturing (LAM) for Aircraft Industry
}

\author{
Claus Emmelmann*a, Maren Petersen ${ }^{\mathrm{a}}$, Jannis Kranz ${ }^{\mathrm{a}}$, Eric Wycisk ${ }^{\mathrm{b}}$ \\ ${ }^{a}$ Institute of Laser and System, Technologies (iLAS), Hamburg University of Technology (HUT), \\ Denickestraße 17 (L), 21073 Hamburg, Germany \\ ${ }^{\mathrm{b}}$ Laser Zentrum Nord GmbH (LZN), Harburger Schlossstr. 6-12, 21079 Hamburg, Germany
}

\begin{abstract}
Today many challenges lie ahead of the aircraft industry. The increasing competition and shortage of resources raise a challenge for future manufacturing technologies and lightweight design. A possibility to cope with these circumstances is the manufacturing technology of Laser Additive Manufacturing (LAM). However there are still challenges to cope with due to the processes novelty, such as the development of further materials, especially lightweight alloys, and new design approaches. Therefore innovative approaches for material development and lightweight design were created in order to fully exploit the processes potentials. The material development process is based on an analytical calculation of temperature distribution versus effective process factors in order to identify acceptable operating conditions for the LAM process. A novel approach to extreme lightweight design was realized by incorporating structural optimization tools and bionic structures into one design process. By consequently following these design principles, designers can achieve lightweight savings in designing new aircraft structure and push lightweight design to new limits.
\end{abstract}

Keywords: Additive Layer Manufacturing; Laser Additive Manufacturing; Selective Laser Melting; lightweight design; bionic design; structural optimization; material development, ANE-Method

\section{INTRODUCTION}

In addition to the already existing American competitors, a rising number of internationally acting companies from Far East and Russia lead to a steadily increasing pressure of cost efficiency and innovation in the European aircraft industry. The increasing struggle for more efficient aircrafts shows, among others, in the rising use of lightweight structures based on structural optimization tools. The introduction of these load optimized components result in falling lot sizes, highly complex parts and the demand for reduced production times at lower raw material charges. Thus manufacturing by conventional processes becomes increasingly more cost-intensive. Therefore a flexible and fast manufacturing process that is able to produce manifold product variants of a high geometric complexity is necessary. A possible process to cope with these challenges is Laser Additive Manufacturing (LAM), see figure 1.

\section{LASER ADDITIVE MANUFACTURING (LAM)}

Laser Additive Manufacturing (LAM) is based on a repeating layer wise manufacturing process which uses a laser beam to melt and solidify material in a powder bed according to slices of a corresponding 3D-CAD model. The stepwise production causes the reduction of complex three dimensional geometries into simple two dimensional manufacturing steps and enables the production of highly complex parts without the need for complex tools [1,2]. The individual production steps of the SLM process are shown in figure 1. In the preprocessing a 3D-CAD-model is divided into horizontal slices with thickness corresponding to the layers in the production process. Typical layer thicknesses for TiAl6V4 are hereby $30 \mu \mathrm{m}-50 \mu \mathrm{m}$. Subsequently to the "slicing" the prepared data is transmitted to the SLM machine in which the actual manufacturing process occurs in three repeating steps. First a powder layer is applied to the base plate and in the second step exposed by the laser beam.

*ilas@tuhh.com; phone +49 4042878 - 3456; fax +49 $40 \quad 42878$ - 4076; http://www.tu-harburg.de/ilas

SPIE Eco-Photonics 2011: Sustainable Design, Manufacturing, and Engineering Workforce Education for a Green Future, edited by Pierre Ambs, Dan Curticapean, Claus Emmelmann, Wolfgang Knapp, Zbigniew T. Kuznicki, Patrick P. Meyrueis, Proc. of SPIE Vol. 8065, 80650L · C 2011 SPIE · CCC code: 0277-786X/11/\$18 - doi: 10.1117/12.898525 
Due to the energy input of the focused laser beam the powder melts and solidifies into welding beads after exposure. In every single layer the beam melts the surface area corresponding to the CAD - model slice. With each new layer the base plate is lowered and powder is applied. After completion of all layers the part can be taken from the powder bed and unmelted powder recycled for further production.

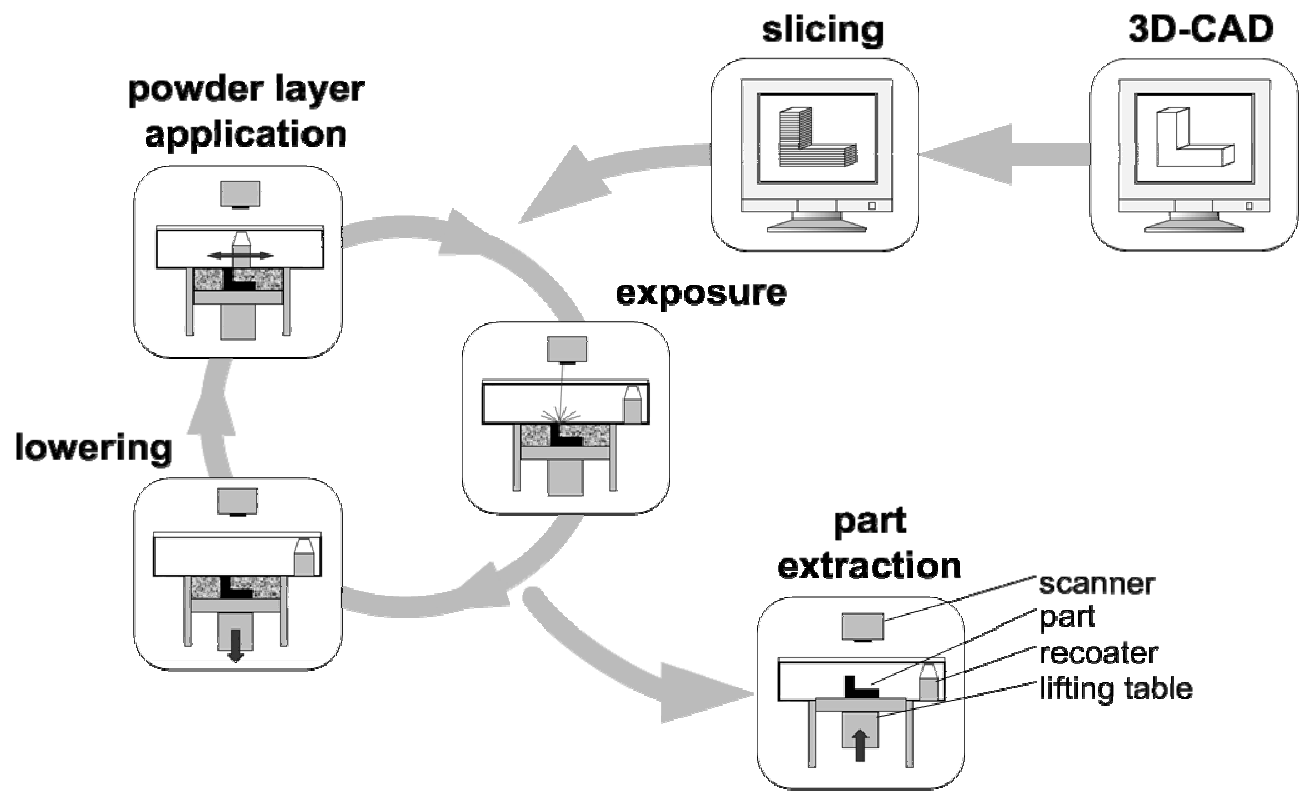

Figure 1. Process of Selective Laser Melting [3].

Besides the ability to manufacture highly complex parts, the high recycling rate of additive manufacturing leads to a reduction of material waste during machining, compared to conventional subtractive manufacturing processes, like shape cutting. Besides the raw material cost savings there is an ease in handling smaller billets of raw feedstock, by-products or scrap produced from a less-extensively machined part. Additional benefits occur thru the tool less manufacturing process by saving tool costs especially when processing high abrasive materials such as titanium.

However, considering restrictions and other additional benefits of the LAM process the assessment for an economical production with LAM is more complex. Possible limitations for economic LAM production and geometrical possibilities are among others high machine hours, high powder costs compared to wrought material, the need for support structures (figure 2), the limitations of the building chamber, limited surface qualities due to powder adhesion and potential need for finishing. Pitted against these negative effects are benefits from new engineering possibilities. LAM allows converting structurally optimized geometries and bionic structures without adaptation into the part design which results in extremely strong lightweight designs (figure 2). Also the integration of additional functions which in conventional designs have to be solved thru multiple part designs can be realized. 

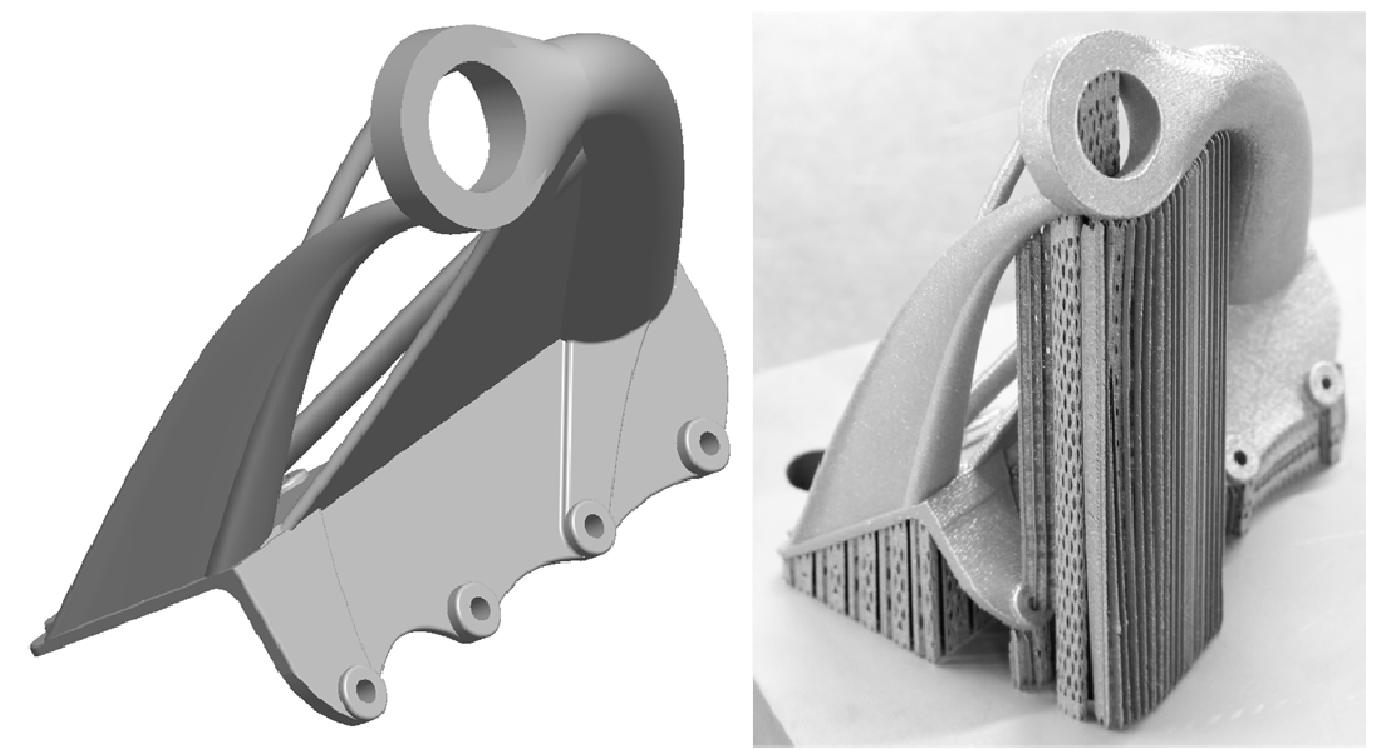

Figure 2. CAD-model of optimized bionic part and its manufactured counterpart with support structures.

\section{PROCESS DEVELOPMENT FOR NEW MATERIALS}

\subsection{Overview}

Currently opposed to the lightweight potential due to the freedom of design is a still limited lineup of materials well suitable for lightweight design. Hence it is essential to qualify further lightweight alloys for LAM and thereby exploiting additional areas of application for the LAM process. However, the qualification of new material systems for LAM is a cost and time consuming process involving extensive experimental effort. The identification of suitable process parameters is an iterative procedure when performed experimentally. Thus, a reliable optimization of this procedure is desirable. Final properties of parts manufactured in the LAM process depend on the operating conditions as well as on the characteristics of the powder material. Optimization of a process with a large number of operating parameters is a complex task. Therefore, a systematic approach should include a simulation to identify a possible process window and a design of experiments with respect to the results of the simulation. The influence of characteristic process variables such as laser power, scan speed, hatch distance can be described theoretically for each material system without the need for experiments [4]. Thus acceptable process conditions can be identified by using the model described in this section.

\subsection{Physical model}

The interaction between the laser beam and the material can be modelled based on the physical phenomena occurring during the process. For LAM processes it is useful to compose three sub-models according to Sun [5], which can be solved sequentially as shown in figure 3. The optical model is the calculation of the heat absorbed in the powder bed which derives from the laser beam parameters like intensity, laser beam distribution and the optical properties of the material. The amount of heat can be used in a heat source function in the thermal sub-model. In this thermal model the heat transfer equations in the powder bed will be set up using the thermal properties of the material and the significant process parameters. The calculated temperature of the powder bed can be used in the sintering sub-model to determine the rheological properties corresponding to these temperature conditions. Although it is clear that the 


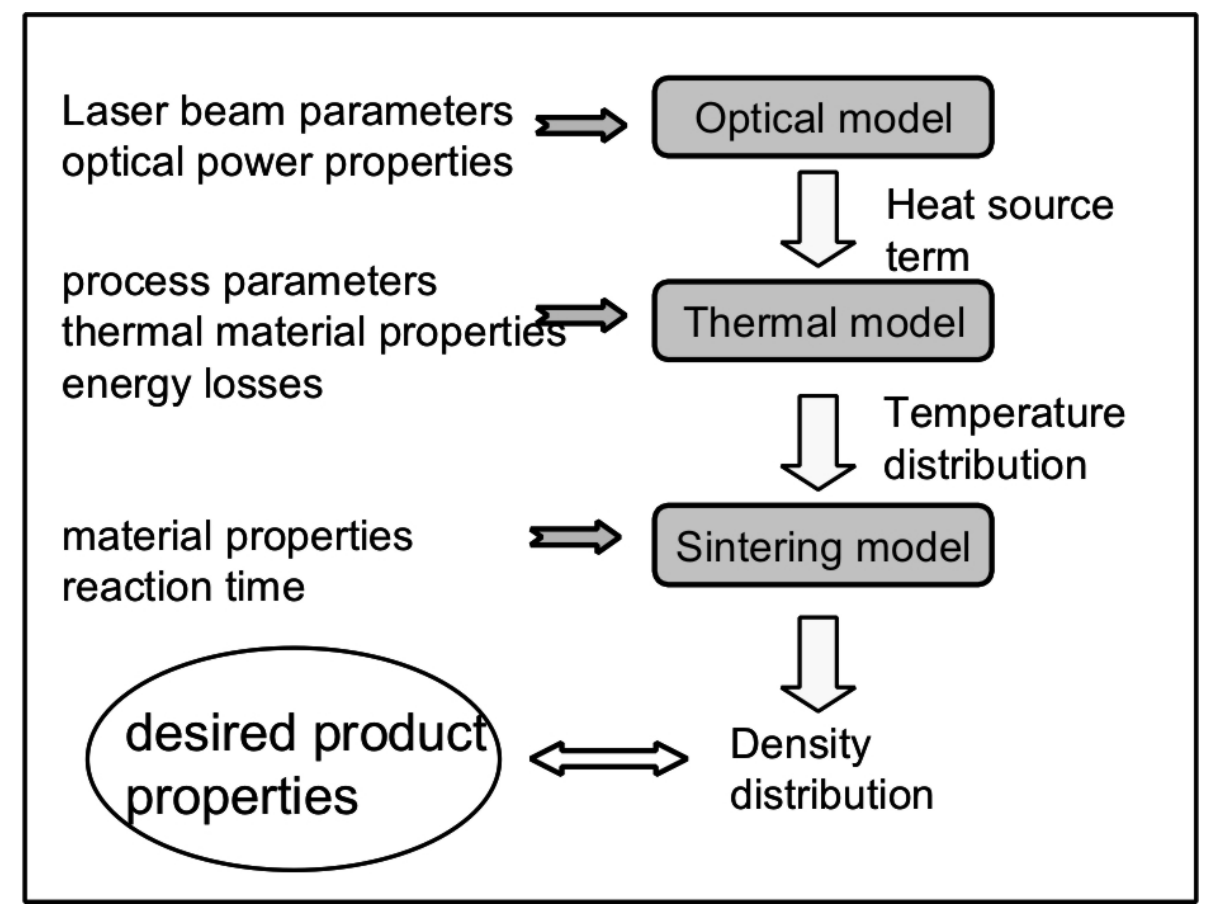

Figure 3. Models required for temperature estimation.

temperature in the zone of interaction between the laser beam and the material takes great influence on the metallic microstructure of a finished part $[3,6]$ the majority of its properties is controlled by part porosity. Thus it is desirable to eliminate the part's porosity by completely remelting the powder material. Objective of the model introduced in this paper is to calculate the temperature distribution in the powder bed during the laser beam exposure process.

\subsection{Thermal model}

The heat source function according to equation 1 is a solution of the average laser intensity distribution. The coordinate system is given in figure 4 and all symbols are given in the appendix. In this solution the cross section intensity profile of the laser beam is described by a Gaussian profile and transmission of heat through the powder bed is neglected such that only absorption and reflection of laser beam energy are considered. Sometimes a rectangular function of the intensity is also used to reduce the calculation time. The effective surface absorptivity given in equation 2 can be calculated or measured (see Appendix for denotations).

$$
\begin{aligned}
\begin{aligned}
q(x, y, z)=\frac{\partial I}{\partial z}= & -(1-R)_{e f f} \beta I_{0} . \\
& e^{\left(-2\left(x^{2}+y^{2}\right) / r_{b}-\beta \cdot z\right)}
\end{aligned} \\
A_{e f f}=(1-R)_{e f f}
\end{aligned}
$$

The evolution of temperature vs. time in the powder bed is usually determined by balancing the energy in the thermal sub-model. Heat transfer in laser processing has generally been a wide area of research. In LAM processes the heat transfer problem is a combination of the three mechanisms of convection, conduction and radiation. On one hand it is possible to describe the process for metallic materials with the equation of effective heat conduction [5]. 
The effective thermal properties of the powder bed must then be determined depending on material and its temperature. On the other hand it is possible to calculate the terms of convection and radiation according to the process parameters and to integrate the mathematical description of these phenomena in the model. Under typical operating conditions the energies of convection and radiation are low compared to the heat source. Hence, the heat transfer problem can be described with a heat conduction approximation only as shown in equation 3.

$$
\nabla(-\lambda \nabla T)=\dot{q}
$$

Although the powder bed cannot be characterized by the thermal properties of a solid material, the thermal conductivity of powder beds may be calculated according to various models given in the literature [7]. In order to test the suitability of materials for LAM it is important to know if the temperature distribution in the powder bed due to the applied laser beam is above the melting point of the powder's lowest melting component. Therefore, the most crucial point in developing this theory is the thermal sub-model with calculation of the temperature distribution at the powder bed surface.

\subsection{Analytical solution}

The temperature distribution at the surface of the powder bed is derived from a one-dimensional model. Thus the suitability of the powder mixture can be evaluated theoretically. An analytical solution of the heat conduction equation (equation 3) is available for specific initial and boundary conditions. For a simplified one-dimensional heat conduction problem where the powder bed is represented by a semi-infinite solid with constant thermal properties, the temperature at the powder bed surface can be calculated easily [8]. With this calculation various modifications of a composite material can be tested for the LAM process and the thermal profiles allow a qualitatively correct prediction of the melting process.

The theoretical surface temperature and the temperature distribution in the powder layer identify a reference value for the process window of the given material system in respect to the laser beam source. This simple one-dimensional model predicts the maximum surface temperature in order to describe the relationship between operating conditions and temperature distribution. The presumptions have to be adjusted to real conditions by a numerical solution.

\subsection{Numerical model}

A numerical solution of the heat conduction equation (equation 3) can be realized by finite element methods (FEM) and a computer simulation. In this context a three-dimensional solution of the temperature distribution is feasible. The melting depth corresponding to the layer thickness can be calculated using the numerical solution of this model. The width of the melting zone of each melt track can by calculated to define the required hatch distance. Some results of the calculation of the temperature at discrete points on the powder bed surface 

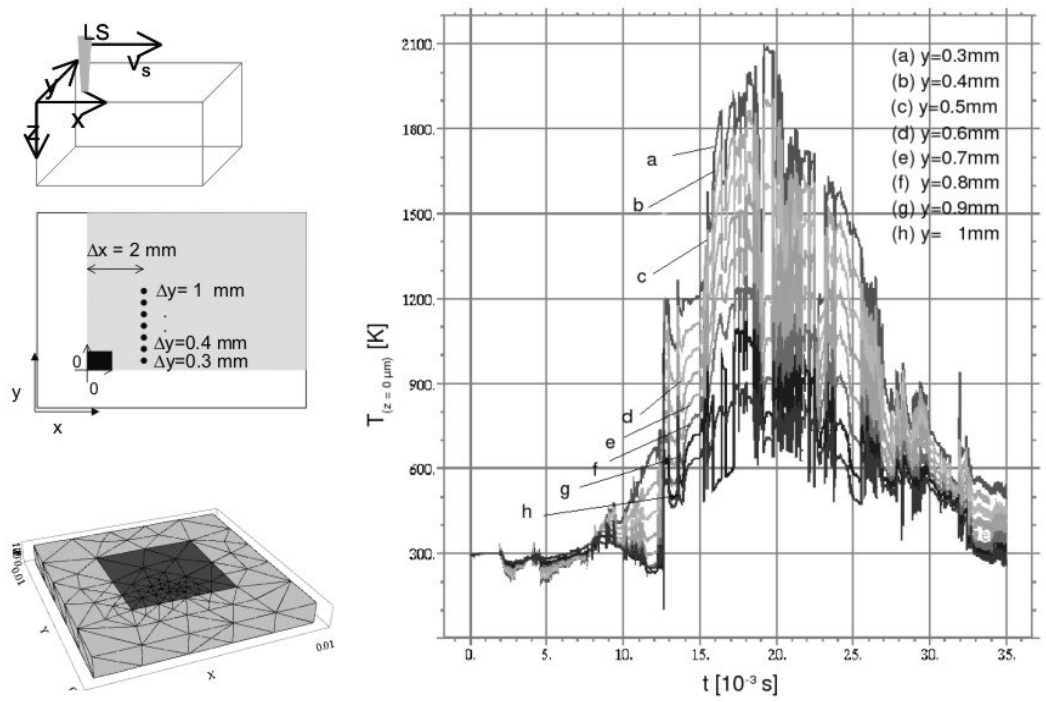

$\mathrm{Ti}, \mathrm{c}_{\mathrm{p}} \lambda, \mathrm{P}=100 \mathrm{~W}, \mathrm{v}_{\mathrm{s}}=100 \mathrm{~mm} / \mathrm{s}, \mathrm{A}_{\text {eff }}=0.5, \mathrm{t}_{1}=0.05 \mathrm{~s}$, $\mathrm{d}_{\mathrm{s}}=0.4 \mathrm{~mm}$, porositity $=0.5$

Figure 4. Results of process modelling for Titanium.

for titanium powder are shown in figure 4. Typical process parameters such as $100 \mathrm{~W}$ of laser beam power and a scan speed of $100 \mathrm{~mm} / \mathrm{s}$ as well as $50 \%$ effective absorptivity and $50 \%$ density of the powder bed were assumed. The obtained results show that titanium can be melted sufficiently with nearly standard process parameters which are used for the processing of e.g. stainless steel. With this simulation the production time of a specified part can also be calculated according to the process parameters of layer thickness and hatch distance in order to gain a prediction for cost and time effectivity of LAM.

\subsection{Experimental qualification}

The results of the simulation depend on the accuracy of the material data. For solids the temperature-dependent values are available in the literature but powder beds or composites require an approximation. The results of determining the temperature distribution depend on the accuracy of the approximation of the effective thermal properties of the powder bed. To verify the processing conditions a statistical design of experiments is necessary to obtain acceptable process parameters for parts with desired properties. The initial parameters of the experiment were carried out with the process parameters which were calculated with the model derived above. Specially designed control parts were used to determine the scan strategy and the desired part properties. This three step method for qualifying material systems for LAM is called ANE (analytical, numerical, experimental) as shown in figure 5 [9]. 


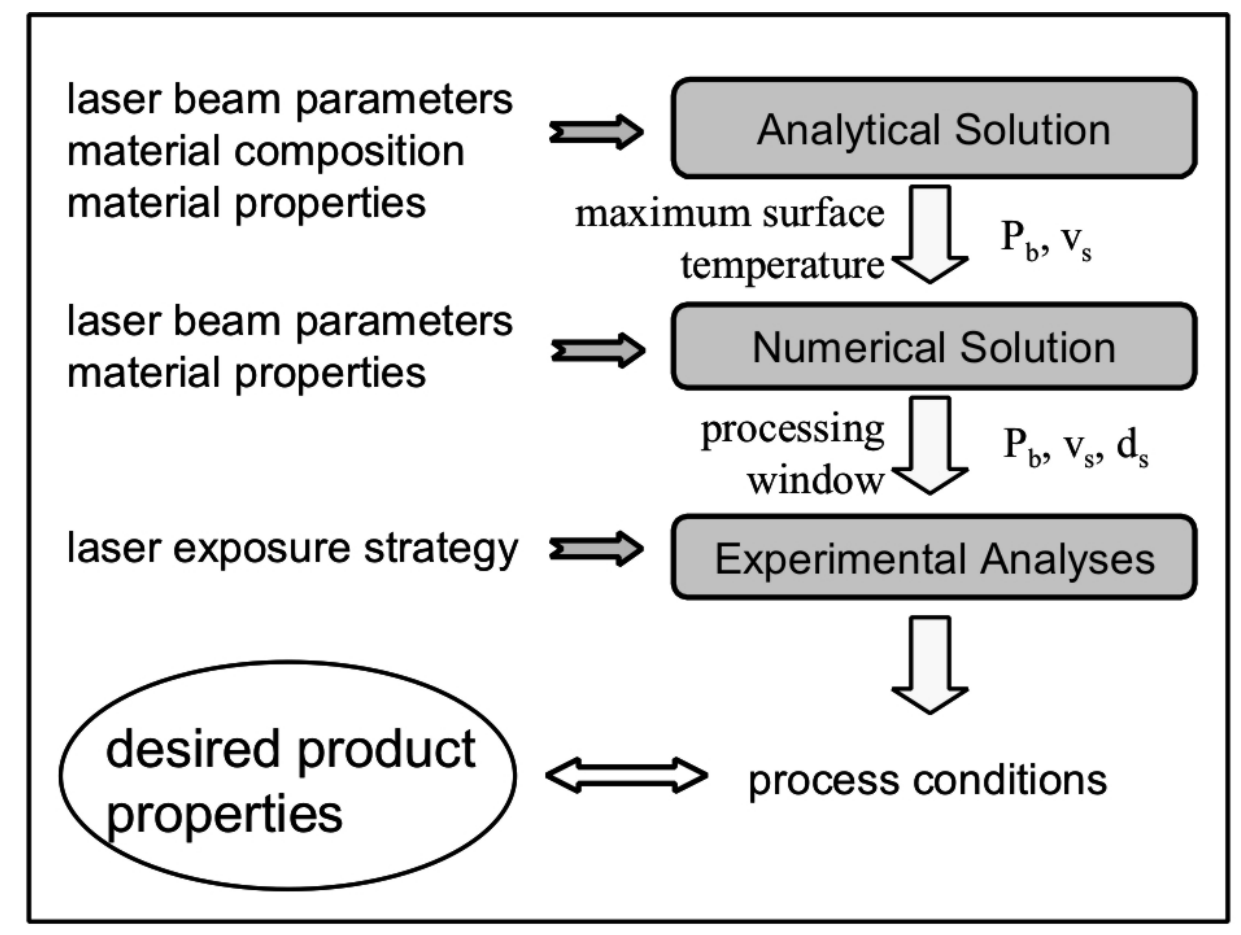

Figure 5. ANE method of process window determination.

With the described material development process on hand it is now possible to significantly expand the available material lineup of lightweight alloys at a reduced expenditure regarding cost and time. But the development of further materials is only one necessity for exploiting LAM processes full potential and to unlock further areas of application. Supplementary to this it is decisively to be able to fully utilize the process inherent freedom of geometry and to facilitate lightweight constructions not possible up to now. Hence an innovative design process approach was developed which will be described in the following chapter.

\section{STRUCTURAL OPTIMIZATION AND BIONICS}

The methods for structural optimization are manifold, see figure 6. Therefore just a brief summary will be given exemplarily with the help of a simple example: a bridge. The "choice of design" includes the consideration of truss construction or compound structures. Furthermore it has to be decided whether the parts design shall be integral or differential. During the "choice of materials" the materials themselves are the design variables which have to be chosen according to the case specific requirements. The preceding methods of structural optimization were of an analytical nature. With the advent of affordable and highly capable personal computers, numerical methods for structural optimization are being increasingly used. The major methods will be described briefly in the following. 


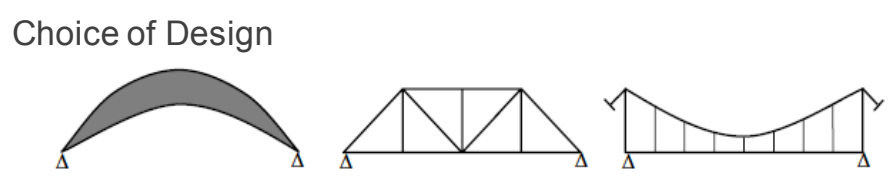

Choice of Material Properties

Aluminium

Topology Optimization

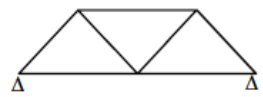

Shape Optimization

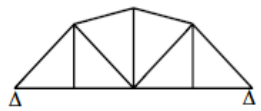

Parameter Optimization

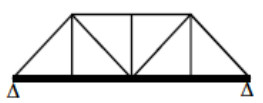

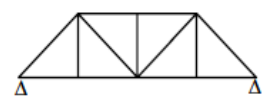
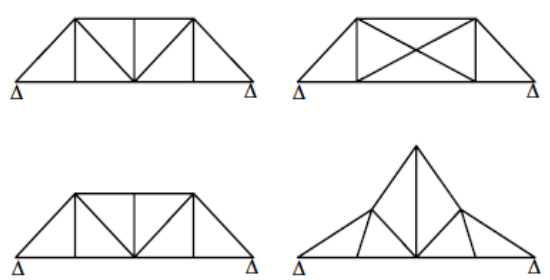

Composites

Steel

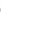


the discretization of design space which includes the modeling of design space and the definition of the FEM-problem (e.g. forces, mesh, constraints, etc.) [11]. The definition of the optimization problem is then carried out by the declaration of design variables, the definition of objective function e.g. weighted compliance or a volume reduction of $85 \%$, and the definition of manufacturing constraints. Thereon an FE-analysis is being performed in order to determine the parts stress distribution. Basis of the optimization algorithm is the adaption of the element density by a fictional material law during the iterative optimization process. Elements of high structural relevance will be assigned a density of $<1$ and elements of negligible structural relevance $>0$. During the break-off criteria examination the model is checked weather the objective functions change lies beneath a specific value, otherwise an further iteration will start. The consequence of this process is a constant evolving of the structural relevant volume during the iterations.
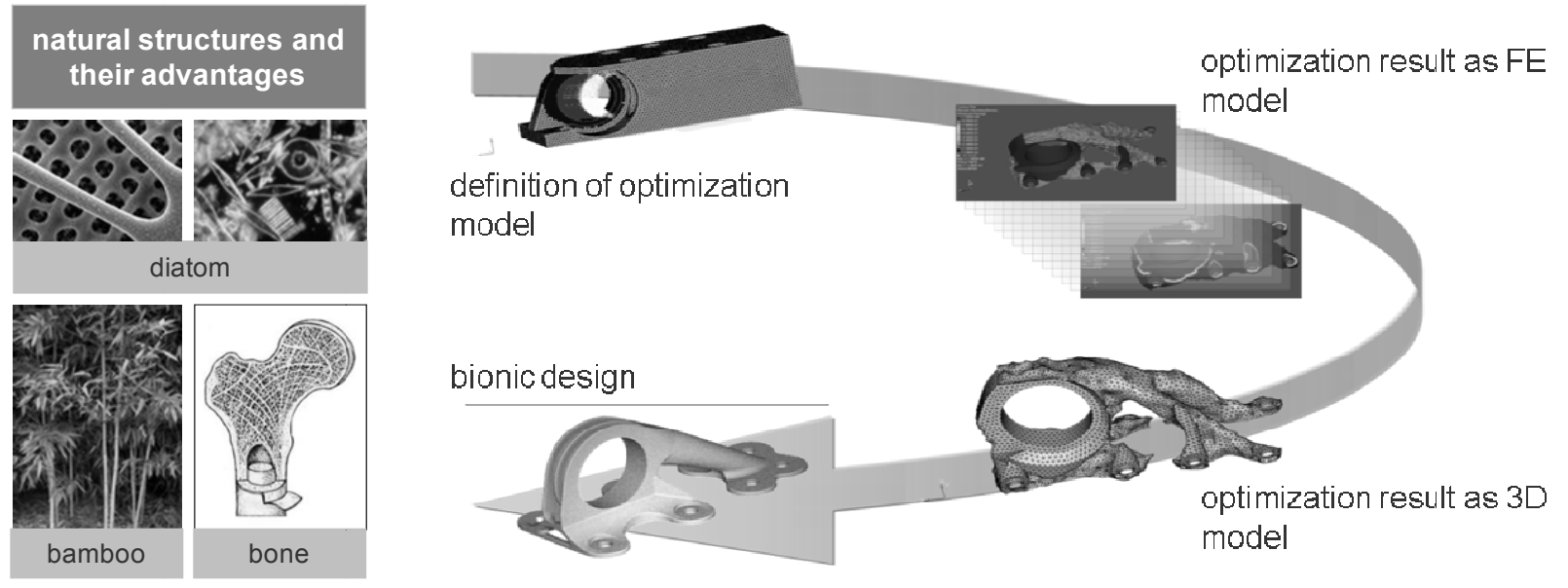

Figure 8. Application of topology optimization and potential bionic lightweight structures.

Figure 8 shows an application of the topology optimization and potential biological lightweight structures. With the help of the topology optimizations implementation in the development process of "design-for-LAM" the following part characteristics can be achieved [12,13]:

- minimization of weight

- reduction of peak stresses

- homogeneous stress distribution

- reduction of production waste

Using the explained methods iLAS optimized the part design and production of an aluminium bracket in an A380 Airbus, see figure 9. This bracket fixes, combined with others, the crew rest compartment to the secondary structure of the airplane. More than 25.000 brackets fulfil similar functions in one aeroplane A380, which most of them are mulled with intensive machining load resp. chipping removal. The incorporation of bionic inspired bamboo-beams facilitated a very stiff and light bracket design that is not manufacturable by any other process, see figure 9. An overall weight reduction of $50 \%$ could be realized due to the design freedom of LAM and a material change to titanium $[12,13]$. 

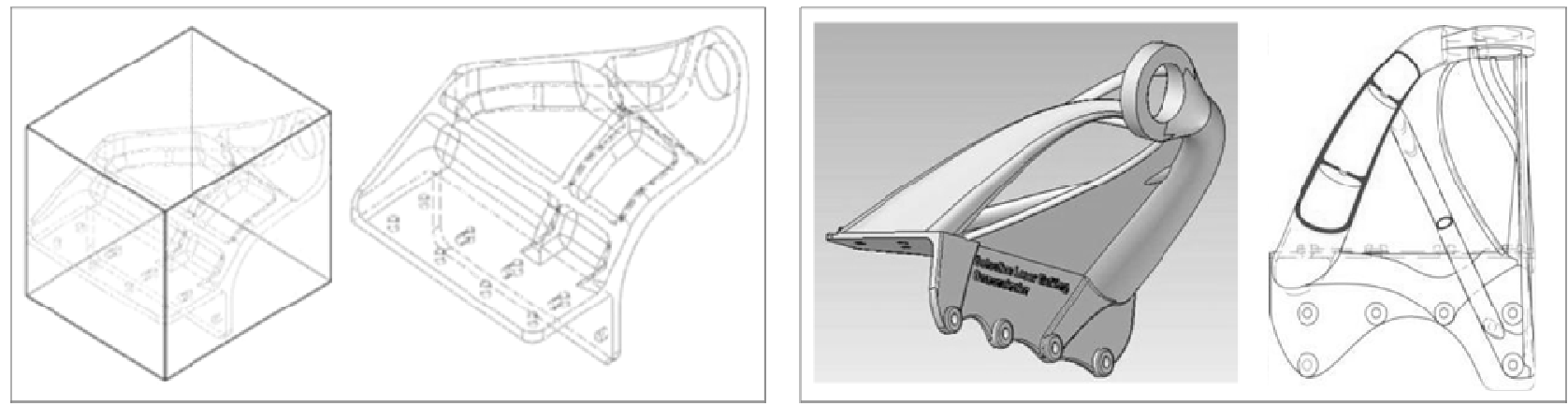

Figure 9. left: Raw part and conventionally manufactured bracket. Right: Topology optimized bracket with bamboo structure [12].

Even though a simple manufacturing process substitution combined with a redesign can achieve a tremendous weight reduction, the LAM potential for lightweight design goes far beyond this and needs far more radical approaches in order to be fully exploited. In addition to bionic and optimized design further lightweight potential could be successfully shown by an integration of functions with the help of another A380 bracket design which was initially directly attached onto the honeycomb, see figure 10 . The force off-set from the neutral axis generated a momentum and additional loads that had to be regarded during the design phase. Aiming for an improved lightweight design and an optimized load input the initial bracket and the honeycomb insert plate were combined, following the bionic analogy of the tree and its load transfer from branches to the trunk, see figure 10. By directly gluing the part into the sandwich compound a direct load induction into the neutral axis is achieved.
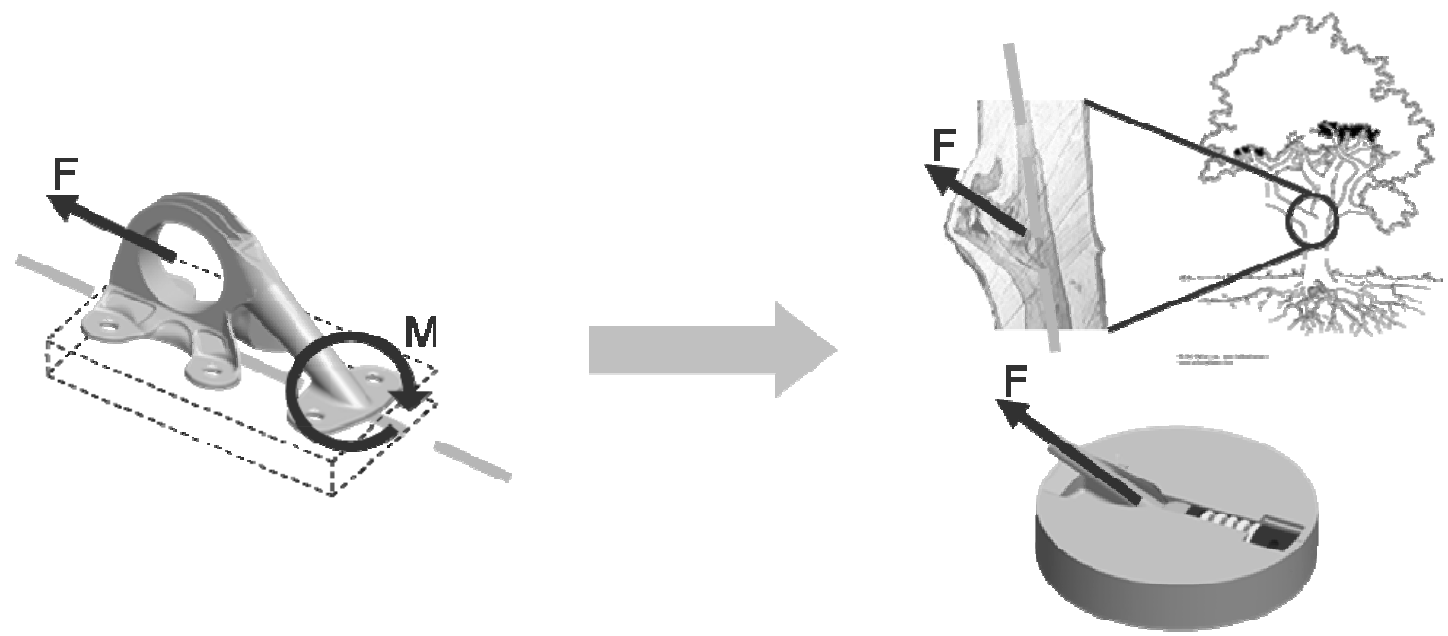

Figure 10. Adapted bracket design incorporating integration of functions and loading description.

Figure 11 summarizes the results of weight saving for the different designs of the analyzed A380 bracket. The initial design was manufactured by milling and weights $330 \mathrm{~g}$, the insert plate including fasteners weights ca. $1.400 \mathrm{~g}$. In comparison the bionic bracket designed for a manufacturing process substitution shows a weight saving of $41 \%$ whereas the radical approach of function integrations even more than $80 \%$. 


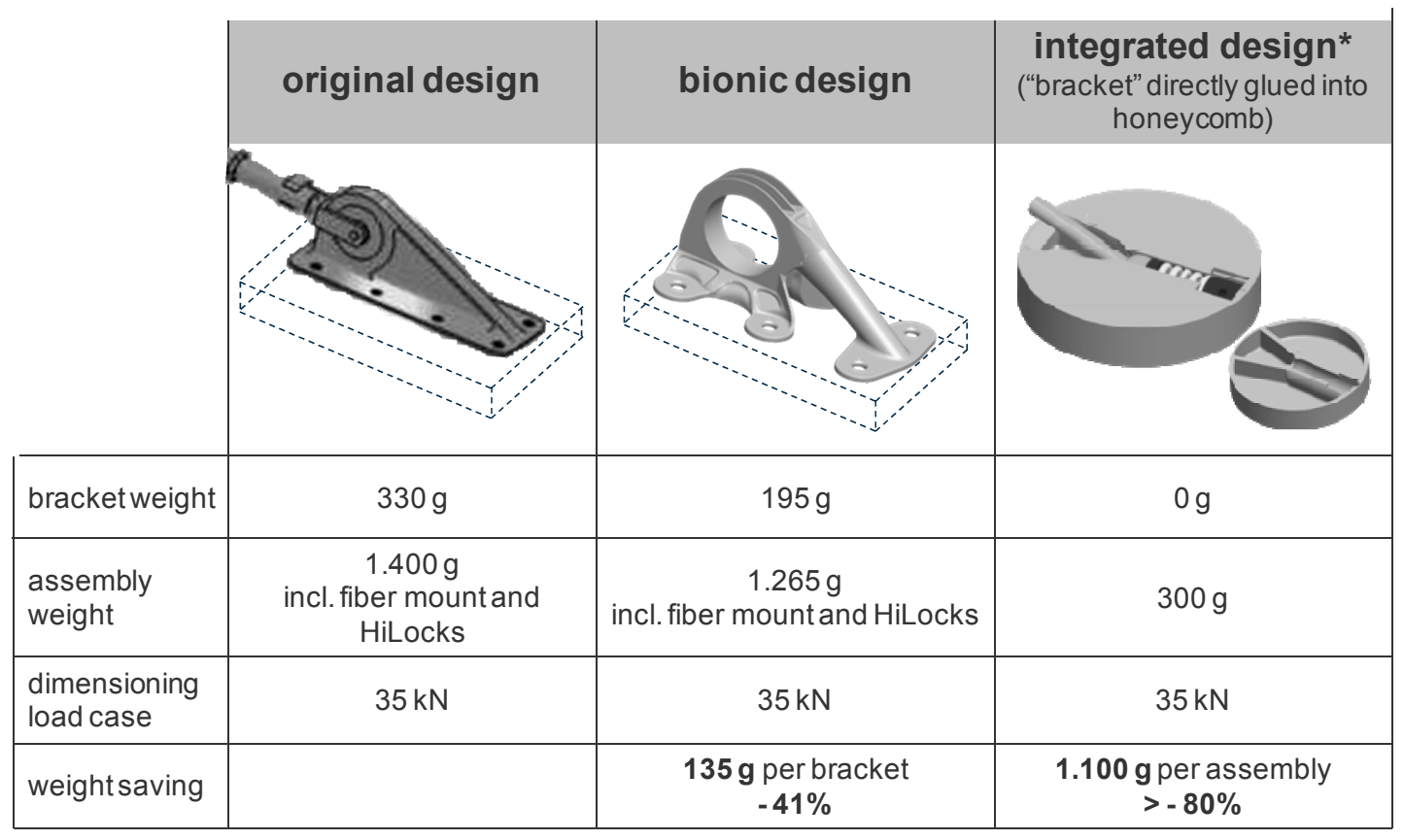

Figure 11. Comparison of different design alternatives of aircraft brackets.

\section{CONCLUSION}

New layer wise manufacturing technologies such as Laser Additive Manufacturing (LAM) allow innovative approaches to product design. Especially for lightweight design in aircraft applications LAM offers new possibilities for loadadapted structures. However the available material range is still limited and new applications often attend the need for qualifying new materials which is a cost and time consuming process involving extensive experimental effort. The identification of suitable process parameters is an iterative procedure when performed experimentally. Using an interactive procedure between a theoretical calculation of the material behaviour with respect to laser radiation and an experimental optimizing of process parameters time and / or costs can be reduced significantly. With the presented mathematical model of the temperature distribution versus effective factors of the process a region of acceptable operating conditions for the laser generating process could be found. This strategy leads to a rather small number of required experiments to develop a new material. This concept is feasible only if the database for the heat capacities and thermal conductivities at the various temperatures of the laser generating process is accurate. For solids the functions are available in the literature, but for powder beds or composites there is an approximation. The results of the temperature determination are only as accurate as possible by the approximation of the thermal properties.

To fully capture lightweight potential of LAM technologies new design guidelines and processes have to be developed. A novel approach to extreme lightweight design is realized by incorporating structural optimization tools and bionic structures into one design process. By consequently following this design process designers can achieve lightweight savings in designing new aircraft structures. Limited by the current 3D-CAD-Tools and the not yet holistic design guidelines for LAM the results of the conducted design optimizations only show the beginning of what can become an essential new way of designing lightweight constructions. 


\section{APPENDIX}

Table 1. Denotations of used symbols and their units.

\begin{tabular}{|l|l|l|}
\hline$\beta$ & effective absorption coefficient & \\
\hline$\delta$ & penetration depth & $\mathrm{mm}$ \\
\hline$\lambda$ & thermal conductivity & $\mathrm{W} / \mathrm{mK}$ \\
\hline$\rho$ & density & $\mathrm{Kg} / \mathrm{mm}^{3}$ \\
\hline$A$ & absorptivity & \\
\hline $\mathrm{c}_{\mathrm{P}}$ & specific heat capacity & $\mathrm{J} / \mathrm{kg} \mathrm{K}$ \\
\hline $\mathrm{I}$ & intensity & $\mathrm{W} / \mathrm{mm}^{2}$ \\
\hline $\mathrm{h}_{\mathrm{s}}$ & hatch space & $\mathrm{mm}$ \\
\hline $\mathrm{q}$ & heat input & $\mathrm{W} / \mathrm{m}^{2}$ \\
\hline $\mathrm{r}_{\mathrm{b}}$ & beam radius & $\mathrm{mm}$ \\
\hline $\mathrm{R}$ & reflectivity & \\
\hline $\mathrm{P}$ & laser power & $\mathrm{W}$ \\
\hline $\mathrm{T}$ & temperature & $\mathrm{K}$ \\
\hline $\mathrm{t}$ & time & $\mathrm{s}$ \\
\hline $\mathrm{V}_{\mathrm{S}}$ & scan velocity & $\mathrm{mm} / \mathrm{s}$ \\
\hline
\end{tabular}

\section{REFERENCES}

[1] Gebhardt, A., "Generative Fertigungsverfahren", Carl Hanser Verlag, München, (2007).

[2] Zäh, M. F.., "Wirtschaftliche Fertigung mit Rapid-Technologien", Carl Hanser Verlag, München, (2006).

[3] Meiners, W., "Direktes Selektives Laser Sintern einkomponentiger metallischer Werkstoffe", Shaker Verlag, Aachen, (1999).

[4] Petersen, M.; Emmelmann, C., "Theoretical and Experimental Studies of Direct Laser Generating of Ceramic Metal Composites", In: Proc. of the $3^{\text {rd }}$ International WLT-Conference on "Lasers in Manufacturing", München, Germany, (2005).

[5] Petersen, M., "Lasergenerieren von Metall-Keramik-Verbundwerkstoffen", $\mathrm{PhD}$ thesis, Hamburg University of Technology, Hamburg, Germany, (2006).

[6] Wagner, C. A., "Untersuchungen zum Selektiven Lasersintern von Metalen", PhD thesis, RWTH Aachen, Aachen, Germany, (2002).

[7] Nelson, J. C., "Selective Laser Sintering: A Definition of the Process and an Empirical Sintering Model", PhD thesis, University of Texas, Austin, USA, (1993).

[8] Carslow, H. S.; Jaeger, J. C., "Conduction of Heat in Solids", Oxford University Press, Oxford, UK, (1959).

[9] Petersen, M.; Emmelmann, C., "Development of New Material Systems for Direct Laser Freeform Fabrication", In: Proc. of ICALEO 2006, Scottsdale, AZ; USA, (2006).

[10] Schumacher, A., "Optimierung mechanischer Strukturen - Grundlagen und industrielle Anwendungen", Springer-Verlag, Berlin, Heidelberg, (2005).

[11]Bendsøe, P.; Sigmund, O., "Topology Optimization: Theory, Methods ans Applications", Springer-Verlag, Berlin, Heidelberg, (2003).

[12] Wycisk, E.; Kranz, J.; Emmelmann, C.; Leichtbaupotenzial durch das Lasergenerieren bionischer Strukturen; RapidTech 2010, (2009).

[13] Kranz, J.; Wycisk, E.; Emmelmann, C.; „RM-gerechte Produktentwicklung von Flugzeugkomponenten für AIRBUS“; 15. Anwenderfoum RPD, Fraunhofer-Institut für Produktionstechnik und Automatisierung IPA, (2010). 